(n)

trobertivier

Journal of Nonlinear Mathematical Physics

ISSN (Online): 1776-0852 ISSN (Print): 1402-9251

Journal Home Page: https://www.atlantis-press.com/journals/jnmp

\title{
Contiguity relations for linearisable systems of Gambier type
}

A. Ramani, B. Grammaticos, P. Guha

To cite this article: A. Ramani, B. Grammaticos, P. Guha (2013) Contiguity relations for linearisable systems of Gambier type, Journal of Nonlinear Mathematical Physics 20:4, 565-576, DOI: https://doi.org/10.1080/14029251.2013.868269

To link to this article: https://doi.org/10.1080/14029251.2013.868269

Published online: 04 January 2021 


\title{
Contiguity relations for linearisable systems of Gambier type
}

\author{
A. Ramani \\ Centre de Physique Théorique, Ecole Polytechnique \\ CNRS, 91128 Palaiseau, France \\ Alfred.Ramani@cpht.polytechnique.fr \\ B. Grammaticos \\ IMNC, Université Paris VII \& XI, CNRS, UMR 8165 \\ Bât. 440, 91406 Orsay, France \\ grammaticos@imnc.in2p3.fr \\ P. Guha* \\ IHES, 35 route de Chartres \\ 91440 Bures sur Yvette, France \\ partha@boson.bose.res.in
}

Received 17 August 2013

Accepted 4 October 2013

\begin{abstract}
We introduce the Schlesinger transformations for the Gambier, linearisable, equation and by combining the former construct the contiguity relations of the solutions of the latter. We extend the approach to the discrete domain obtaining thus the Schlesinger transformations and the contiguity relations of the solutions of the Gambier mapping. In all cases the resulting contiguity relation is a linearisable equation, involving free functions, and which can be related to the generic Gambier mapping.
\end{abstract}

Keywords: contiguity relations; linearisability; Gambier equation.

PACS numbers: 02.30.Ik, 05.45.Yv

\section{Introduction}

Contiguity relations play a very important role in integrable systems. They extend to the nonlinear domain the various relations which exist for the hypergeometric function and its degenerate forms. A contiguity relation (in the sense we shall use throughout this paper) is a non-differential recursion relation of a function with respect to a parameter at a fixed value of the independent variable. Moreover, as one would expect from the use of the term contiguity, these are relations between neighbouring values of the parameter. Typically, for the equations of the hypergeometric family this is a three-point recursion involving values of the parameter differing by \pm 1 . We can illustrate this in the case of the Bessel function $Z_{\alpha}$ for which the well-known recursion [1] can be written as

$$
Z_{\alpha+1}(t)+\frac{2 \alpha}{t} Z_{\alpha}(t)+Z_{\alpha-1}(t)=0
$$

*Permanent address: Satyendranath Nath Bose National Centre for Basic Sciences, JD Block, Sector III, Kolkata 700098, India . 
It goes without saying that, if the function has several parameters, the contiguity relations may become more involved, relating the values of the function along different directions in the parameter space.

In the case of nonlinear integrable systems, provided they depend on some parameter, it is also possible to establish contiguity relations of the solution. The Painlevé equations are the prototypical example in this case. Let us illustrate this with the example of Painlevé II:

$$
x^{\prime \prime}=2 x^{3}+t x+\alpha
$$

The contiguity relation of its solutions has been known for quite some time. Indeed Jimbo and Miwa [2] have shown that $x_{\alpha}(t)$ satisfies a nonlinear recursion relation with respect to the parameter $\alpha$ of the form

$$
\frac{\alpha+1 / 2}{x_{\alpha+1}+x_{\alpha}}+\frac{\alpha-1 / 2}{x_{\alpha}+x_{\alpha-1}}=2 x_{\alpha}^{2}+t
$$

The most interesting result is that these contiguity relations of continuous Painlevé equations are Painlevé equations on their own right: they indeed define a large class of what are called today the discrete Painlevé equations [3]. This being so, the discrete Painlevé equations do possess contiguity relations themselves. It turned out that in most cases the discrete Painlevé equations have the property of self-duality [4]: the contiguity relation of some discrete Painlevé equations leads back to the same equation. This is in fact true not only for those discrete Painlevé equations which are obtained from continuous ones but for almost all the others, with two notable exceptions, the equations which in the Sakai classification [5] are related to the affine Weyl groups $A_{2}^{(1)} \times A_{1}^{(1)}$ and $A_{1}^{(1)} \times A_{1}^{(1)}$ (although a partial self-duality is possible).

Linearisable equations constitute a substantial part of the integrable family and thus it is natural to wonder whether their solutions may possess contiguity relations. The difficulty in this case is that the freedom in linearisable nonlinear equations corresponds to the appearance of arbitrary functions of the independent variable and not to parameters. However an important exception does exist, and in fact it concerns the "master" linearisable equation among second-order integrable differential equations i.e. the one commonly referred to as the Gambier equation [6]. As we have shown in [7] it is possible to derive Schlesinger transformations for the Gambier equation, relating solutions corresponding to different values of a parameter. The latter is traditionally taken to be integer (but see our comments at the end of section 2). In [7] we have shown that two different Schlesinger transformations do exist corresponding to $\Delta n=1$ and $\Delta n=2$. Unfortunately the result in the $\Delta n=1$ case turned out to be not very convenient for the derivation of the contiguity relation. Here we repeat the derivation and obtain for $\Delta n=1$ a result in parallel to the one for $\Delta n=2$. In section 2 we construct the contiguity relations of the Gambier equation and show that, as expected, they lead to linearisable discrete equations. In [7] we have also derived Schlesinger transformations for the discrete Gambier equation, the Gambier mapping [8]. Again the derivation had to be repeated here since our derivation in [7] was performed under the requirement of integrability which puts constraints on the parameters of the mapping. As we shall explain in what follows, the property of integrability is immaterial for the derivation of the Schlesinger transformations: the only thing that counts is the singularity structure. In section 3 we derive the Schlesinger transformations, for a parametrisation of the Gambier mapping more convenient than the one used in [7], and present the construction of the contiguity relation, obtaining again an equation belonging to the same class of linearisable equations, i.e. a Gambier mapping. 


\section{Constructing the contiguity relations for the Gambier equation}

Before proceeding to the case of the Gambier equation let us sketch the derivation of a contiguity relation [9] in the case of Painlevé equations. The same procedure will be applied mutatis mutandis to the Gambier case. The ineluctable ingredient for the derivation of a contiguity relation is a Schlesinger transformation which allows, starting from a solution $x(t, \alpha)$, where $\alpha$ is a parameter, to construct the solution for $x(t, \alpha+1)$, where the parameter has increased by one unit. Next, one uses some symmetry of the equation which allows to related $x(t, \alpha)$ to $x(t,-\alpha)$. Combining this to the Schlesinger one can construct the solution for $x(t, \alpha-1)$. Let illustrate this in the case of $\mathrm{P}_{\mathrm{II}}$. We have

$$
x(t, \alpha+1)=-x(t, \alpha)-\frac{\alpha+1 / 2}{x^{\prime}(t, \alpha)+x^{2}(t, \alpha)+t / 2}
$$

and

$$
x(t,-\alpha)=-x(t, \alpha)
$$

which leads to

$$
x(t, \alpha-1)=-x(t, \alpha)-\frac{\alpha-1 / 2}{-x^{\prime}(t, \alpha)+x^{2}(t, \alpha)+t / 2}
$$

Eliminating $x^{\prime}$ between (4) and (6) gives precisely the contiguity relation (3).

The Gambier equation is a system of two Riccati equations in cascade. It is usually written as

$$
\begin{aligned}
& y^{\prime}=-y^{2}+b y+c \\
& x^{\prime}=a x^{2}+n x y+d
\end{aligned}
$$

where $n$ is integer. (In [7] we have shown that we can always put $b=0$ by a transformation of the independent variable. In this paper we prefer to keep $b$ since its elimination does not bring any substantial simplification). Eliminating $y$ we obtain

$x^{\prime \prime}=\frac{n-1}{n} \frac{x^{\prime 2}}{x}-\left(\frac{n-2}{n} \frac{d}{x}-b-a x \frac{n+2}{n}\right) x^{\prime}-\frac{a^{2}}{n} x^{3}-\left(a b-a^{\prime}\right) x^{2}+\left(n c-\frac{2 d a}{n}\right) x-d b+d^{\prime}-\frac{d^{2}}{n x}$

Thus a Schlesinger transformation for the Gambier equation will relate two solutions of (7) corresponding to different values of $n$ and, as we have shown in [7], we have two different transformations, with $\Delta n=1$ and $\Delta n=2$. Before proceeding to the transformations a remark is in order concerning the equivalent of (5). It is straightforward to check that the following property holds true

$$
x(-n, a, d)=\frac{1}{x(n,-d,-a)}
$$

i.e. in order to obtain the solution for $n$ we must invert the solution for $-n$ but at the same time permute $a, d$ and change their sign. Given the form of (7) we expect the Schlesinger transformation 


\section{A.Ramani, B.Grammaticos and P.Guha}

to be of the form

$$
\bar{x}=\frac{\alpha x y+\beta x+\gamma y+\delta}{\eta x y+\theta x+\kappa y+\lambda}
$$

(Normally a Schlesinger transformation of (8) should have been expressed in terms of $x$ and $x^{\prime}$. However we can always, using (7b), simplify the expression by eliminating $x^{\prime}$ through the introduction of $y$ ). The bar notation, $\bar{x}$, is introduced in order to indicate that $x$ is a solution of the Gambier equation where the parameter $n$ has been shifted by an amount $\Delta n$ (and the remaining parameters modified in the appropriate way). This means that $\bar{x}$ must satisfy an equation of the form

$$
\bar{x}^{\prime}=\bar{a} \bar{x}^{2}+\bar{n} \bar{x} y+\bar{d}
$$

where $\bar{n}=n+\Delta n$. It is easy to check that we have always $\alpha \eta=0$ and $\gamma \kappa=0$. Moreover the term $x y$ cannot be present simultaneously with the term $y$. We are thus left with four possible forms for $\bar{x}$ :

$$
\frac{\beta x+\delta}{\theta x+\kappa y+\lambda}, \quad \frac{\beta x+\delta}{\eta x y+\theta x+\lambda}, \quad \frac{\beta x+\gamma y+\delta}{\theta x+\lambda} \text { and } \frac{\alpha x y+\beta x+\delta}{\theta x+\lambda},
$$

It is straightforward to check that starting from the expression

$$
\bar{x}=\frac{\tilde{\beta} x+\tilde{\delta}}{\tilde{\theta} x+\tilde{\kappa} y+\tilde{\lambda}}
$$

one can obtain the remaining three by inverting $x$ or $\bar{x}$ or both. We have introduced the "tilde" notation in (12) mainly for cosmetic reasons. The coefficients in (12) relate $x$ to $\bar{x}$ (which correspond respectively to parameters $n$ and $n+\Delta n$ ) and can be understood as lying "half-way" between the two. This will allow more symmetric expressions when the down-shifted (i.e. corresponding to $\underline{n}=n-\Delta n) x, \underline{x}$, is introduced in what follows: the corresponding coefficients will be accompanied by a down-tilde.

We now pursue our analysis with expression (12). Two cases can be distinguished here: either $\tilde{\beta}=0$ in which case we can take $\tilde{\delta}=1$ by division, or $\tilde{\beta} \neq 0$ in which case we can put $\tilde{\beta}=1$. These two cases define indeed two different Schlesinger transformations corresponding to $\Delta n=2$ and $\Delta n=1$ respectively.

Let us start with the former. Substituting

$$
\bar{x}=\frac{1}{\tilde{\theta} x+\tilde{\kappa} y+\tilde{\lambda}}
$$

into (11) we find that the parameters of (13) must be chosen as

$$
\tilde{\theta}=-\frac{a}{\bar{d}}, \quad \tilde{\kappa}=-\frac{n+1}{\bar{d}}, \quad \tilde{\lambda}=\frac{1}{2 \bar{d}}\left(\frac{\bar{d}^{\prime}}{\bar{d}}-\frac{a^{\prime}}{a}\right)
$$

whereupon we find that

$$
\bar{n}=n+2
$$

and $\bar{a}, \bar{d}$ are given by

$$
\bar{n} \frac{\bar{d}^{\prime}}{\bar{d}}=2 b(n+1)-n \frac{a^{\prime}}{a}
$$




$$
2 \bar{d} \bar{a}=2 d a+2 c(n+1)+\frac{a^{\prime \prime}}{a}-\frac{3}{2} \frac{a^{\prime 2}}{a^{2}}-\frac{\bar{d}^{\prime \prime}}{\bar{d}}+\frac{3}{2} \frac{\bar{d}^{\prime 2}}{\bar{d}^{2}}
$$

Having obtained $\bar{x} \equiv x_{n+2}$ we must now compute $\underline{x} \equiv x_{n-2}$. We start from the expression (13), invert both $x$ and $\bar{x}$ and down-shift the parameters in $n$ leading to

$$
\underline{x}=\frac{1}{\dot{\theta}}\left(\frac{1}{x}-\underset{\sim}{\kappa} y-\underset{\sim}{\lambda}\right)
$$

We have obviously

$$
\underline{n}=n-2
$$

while for the parameters of the Schlesinger we find readily

$$
\underset{\sim}{\theta}=-\frac{a}{d}, \quad \underset{\sim}{\kappa}=-\frac{n-1}{d}, \quad \underset{\sim}{\lambda}=\frac{1}{2 d}\left(\frac{d^{\prime}}{d}-\frac{\underline{a}^{\prime}}{\underline{a}}\right)
$$

where $\underline{a}, \underline{d}$ are given by

$$
\begin{gathered}
\underline{n} \frac{\underline{a}^{\prime}}{\underline{a}}=2 b(n-1)-n \frac{d^{\prime}}{d} \\
2 \underline{d} \underline{a}=2 d a-2 c(n-1)-\frac{\underline{a}^{\prime \prime}}{\underline{a}}+\frac{3}{2} \frac{\underline{a}^{2}}{\underline{a}^{2}}+\frac{d^{\prime \prime}}{d}-\frac{3}{2} \frac{d^{\prime 2}}{d^{2}}
\end{gathered}
$$

Thanks to the above relations all parameters of the two Schlesinger transformations can be expressed in terms of the four parameters of the Gambier equation. The contiguity relation is now obtained by eliminating $y$ between (13) and (18). We find

$$
x_{n+2} x_{n-2}+x_{n+2}\left(f_{n} x_{n} / g_{n+2}-h_{n}+g_{n} / x_{n}\right)+f_{n}=0
$$

where the $f, g, h$ are functions of the independent variable (which is now $n$ ) obtained from the $\theta, \kappa, \lambda$ :

$$
f=\frac{\underset{\sim}{\kappa}}{\underset{\tilde{\kappa} \theta}{\sigma}}, \quad g=-\frac{1}{\underset{\sim}{\theta}}, \quad h=\underset{\sim}{\lambda} g+\tilde{\lambda} f
$$

Given the relation of (23) to (13) and (18) its integration is obvious. Once $x$ and $\underline{x}$ are given we use (18) to compute $y$ whereupon (13) becomes a homographic mapping between $x$ and $\bar{x}$. A study of the degree growth [10] of some initial condition of (23) using algebraic entropy technique leads, quite expectedly, to a degree sequence $0,1,2,3,4,5,6, \ldots$ i.e. a linear growth with step 1 , in perfect agreement [11] to the fact that (23) is a Gambier mapping. At this point it is also interesting to perform a small experiment by requiring that the more general mapping $x_{n+2} x_{n-2}+x_{n+2}\left(k_{n} x_{n}+h_{n}+\right.$ $\left.g_{n} / x_{n}\right)+f_{n}=0$ have the same degree growth as (13). We obtain readily the constraint $k_{n}=f_{n} / g_{n+2}$ which is thus the linearisability condition of (23). 
We turn now to the second, $\Delta n=1$, Schlesinger transformation. We start from

$$
\bar{x}=\frac{x+\tilde{\delta}}{\tilde{\theta} x+\tilde{\kappa} y+\tilde{\lambda}}
$$

and substituting into (11) we obtain

$$
\tilde{\boldsymbol{\theta}}=\frac{a \tilde{\kappa}}{n+1}, \quad \tilde{\kappa}=-\frac{n \tilde{\delta}}{\bar{d}}, \quad \tilde{\lambda}=\frac{n}{(n-1) \bar{d}}\left(\tilde{\delta} \frac{\bar{d}^{\prime}}{\bar{d}}-b \tilde{\delta}+d\right)
$$

and

$$
\bar{n}=n+1
$$

The parameters of the equation are given by

$$
(n+1) \bar{a} \bar{d}=(n+2) a\left(d+\tilde{\boldsymbol{\delta}}^{\prime}\right)+n \tilde{\boldsymbol{\delta}}\left(a^{\prime}-a b\right)+a^{2} \tilde{\delta}^{2} \frac{n+2}{n+1}
$$

and the relation

$$
\frac{\bar{d}^{\prime}}{\bar{d}}=\frac{n-1}{n} \frac{\tilde{\delta}^{\prime}}{\tilde{\delta}}-\frac{d}{n \tilde{\delta}}+\frac{(n-1) a \tilde{\delta}}{n(n+1)}+b
$$

while we find that $\tilde{\delta}$ must be a solution of the Gambier equation

$\tilde{\delta}^{\prime \prime}=\frac{n-1}{n} \frac{\tilde{\delta}^{\prime 2}}{\tilde{\delta}}+\left(\frac{n-2}{n} \frac{d}{\tilde{\delta}}+b-a \tilde{\delta} \frac{n+2}{n}\right) \tilde{\delta}^{\prime}-\frac{a^{2}}{n} \tilde{\delta}^{3}+\left(a b-a^{\prime}\right) \tilde{\delta}^{2}+\left(n c-\frac{2 d a}{n}\right) \tilde{\delta}+d b-d^{\prime}-\frac{d^{2}}{n \tilde{\delta}}$

Having obtained $\bar{x} \equiv x_{n+1}$ we proceed to compute $\underline{x} \equiv x_{n-1}$. As in the $\Delta n=2$ case we start from (25), solve for $x$ and down-shift the expression, whereupon we find

$$
\underline{x}=\frac{\underset{\sim}{\delta}+x(\underset{\sim}{\kappa} y+\underset{\sim}{\lambda})}{1-\underset{\sim}{\theta} x}
$$

By substituting into (11) we obtain

$$
\underset{\sim}{\delta}=-\frac{d \underset{\kappa}{\kappa}}{n-1}, \quad \underset{\sim}{\kappa}=\frac{n \underset{\tilde{a}}{\theta}}{\underline{a}}, \quad \underset{\sim}{\lambda}=\frac{n}{(n-1) \underline{a}}\left(\underset{\sim}{\theta} \frac{\underline{a}^{\prime}}{\underline{a}}-b \underset{\sim}{\theta}+a\right)
$$

and

$$
\underline{n}=n-1
$$

The parameters of the down-shifted equation are given by

$$
(n-1) \bar{a} \bar{d}=(n-2) a d-n \underset{\sim}{\theta}\left(d^{\prime}-b\right)-d^{2} \underset{\sim}{\theta^{2}} \frac{n-2}{n-1}
$$

and

$$
\frac{\underline{a}^{\prime}}{\underline{a}}=\frac{n+1}{n} \underset{\sim}{\underset{\theta}{\theta}}+\frac{\theta^{\prime}}{n \underset{\sim}{\theta}}-\frac{(n+1) d \underset{\tilde{\theta}}{\theta}}{n(n-1)}+b
$$


Finally $\underset{\sim}{\theta}$ must be a solution of the Gambier equation

${\underset{\sim}{\theta}}^{\prime \prime}=\frac{n+1}{n} \underset{\sim}{\underset{\sim}{\theta}} \underset{\underbrace{\prime 2}}{\theta}-\left(\frac{n-2}{n} \underset{\sim}{\underset{\theta}{\theta}}-b-a \underset{\sim}{\theta} \frac{n+2}{n}\right) \underset{\sim}{\theta^{\prime}}-\frac{d^{2}}{n}{\underset{\sim}{\theta}}^{3}+\left(d b-d^{\prime}\right) \underset{\sim}{\theta^{2}}-\left(n c-\frac{2 d a}{n}\right) \underset{\sim}{\theta}+a b-a^{\prime}-\frac{a^{2}}{n \underset{\sim}{\theta}}$

By comparing (30) and (36) to (8) we conclude that $-\tilde{\delta}$ and $1 / \underset{\sim}{\theta}$ satisfy the same Gambier equation as $x$.

In order to obtain the contiguity relation in the $\Delta n=1$ case we eliminate $y$ between (25) and (31). We find

$$
x_{n+1} x_{n-1}\left(g_{n}+1 / x_{n}\right)+x_{n+1}\left(g_{n+1} f_{n} x_{n}+h_{n}+k_{n-1} / x_{n}\right)+f_{n}\left(x_{n}+k_{n}\right)=0
$$

where

$$
f=-\underset{\tilde{\tilde{\kappa}}}{\tilde{\kappa}}, \quad g=-\underset{\sim}{\theta}, \quad h=\underset{\sim}{\lambda}-\tilde{\lambda} f, \quad k=\tilde{\delta}
$$

Just as in the $\Delta n=2$ case equation (37) is linearisable and its integration is obvious using (25) and (31). The degree growth of some initial condition, confirms the Gambier character of the equation since it leads to the sequence $0,1,2,3,4,5,6, \ldots$ Generalising (37) to $x_{n+1} x_{n-1}\left(g_{n}+1 / x_{n}\right)+$ $x_{n+1}\left(j_{n} x_{n}+h_{n}+l_{n} / x_{n}\right)+f_{n}\left(x_{n}+k_{n}\right)=0$ and requiring that the degree growth be exactly the same as for (37) leads to the constraints $j_{n}=f_{n} g_{n+1}$ and $l_{n}=k_{n-1}$ i.e. precisely the form of (37).

A few remarks are in order here before proceeding to the examination of the discrete case. First, in all our analysis above we did not make any reference to the Painlevé property. Indeed, as is well known [8] requiring that the Gambier equation possess the Painlevé property puts constraints on the functions $a, b, c, d$. However these constraints are not necessary for the derivation of the Schlesinger transformation and thus for the contiguity relation. The integrable character of the latter does not depend on the singularities of the solution of $x$ in the complex plane of the continuous variable. Carrying this argument one step further we claim that there is no need for $n$ to be an integer (a Painlevé property prerequisite). The only requirement for the contiguity relation is that $\Delta n$ be integer. In fact, we could have taken for $n$ an arbitrary function of $t$ and the main conclusions would have been the same but we preferred to keep $n$ constant in our derivation so as not to overburden the calculations unnecessarily.

\section{Contiguity relations for the Gambier mapping}

The discrete version of the Gambier equation has been proposed and studied from the point of view of singularity confinement in [8] and in [12]. The main idea behind the discretisation is to follow the Gambier construction setting up the mapping as two (discrete) Riccati equations in cascade. Thus the Gambier mapping can be presented as a system of two homographic mappings where the solution of the first one enters linearly in the coefficients of the second one. Given that a full homographic freedom does exist for the variables of both mappings one can wonder whether an arrant simplification of one of the mappings would not simplify the task at hand. This is the attitude we shall adopt in what follows. We shall work with a Gambier mapping of the form

$$
\begin{gathered}
y_{n}=y_{n-1} \\
x_{n+1}=\frac{x_{n}\left(a_{n} y+b_{n}\right)+c_{n} y+d_{n}}{x_{n}\left(f_{n} y+g_{n}\right)+h_{n} y+k_{n}}
\end{gathered}
$$




\section{A.Ramani, B.Grammaticos and P.Guha}

where (39a) means that $y$ is simply a constant and we have assumed a generic form for $x$ (one of the 8 functions can be set to unity by simple division). In what follows we shall be interested in the singularities of $x$ due to $y$. From (39b) we find readily that $x_{n+1}$ is independent of $x_{n}$, something we refer to as "loss of the memory of the initial condition", whenever

$$
\left(a_{n} y+b_{n}\right)\left(h_{n} y+k_{n}\right)-\left(f_{n} y+g_{n}\right)\left(c_{n} y+d_{n}\right)=0
$$

Since (40) is quadratic for $y$ a singularity for $x$ appears when $y$ happens to take the value of one of the two roots of (40), which we represent formally as $\phi_{n}$ and $\psi_{n}$. Next we simplify (39b) using the homographic freedom for $x$. We finally obtain

$$
x_{n+1}=\frac{x_{n}\left(p_{n} y+r_{n}\right)+q_{n}\left(y-\psi_{n}\right)}{x_{n}\left(y-\phi_{n}\right)}
$$

This precise choice of (41) means that if for some $n$ we have $\phi_{n}=y$ and $x_{n}$ is regular, $x_{n+1}$ is infinite and the subsequent $x$ s have a "fixed" value, by which we mean that it is independent of the initial condition. On the other hand if $x_{n}$ happened to be exactly equal to $-q_{n}\left(\phi_{n}-\psi_{n}\right) /\left(p_{n} \phi_{n}+r_{n}\right)$ (which means that the values of all previous $x$ s are "fixed") then for $x_{n+1}$ we would find an indeterminate form of $0 / 0$ type which would allow to introduce a degree of freedom akin to an initial condition. Similar conclusion can be drawn for the singularity at $\psi_{n}$. If for some $n$ we have $\psi_{n}=y$ and $x_{n}$ is generic, the subsequent $x \mathrm{~s}$ are fixed. However if it happens that $x_{n}=0$ then for $x_{n+1}$ we find an indeterminate form allowing to introduce a degree of freedom.

The two singularity-related quantities $\phi_{n}$ and $\psi_{n}$ need not be related in any way. However, if it happens that $\phi_{n}=\psi_{n+N}$ and this common value is precisely that of $y$, then we can have the following singularity pattern. The variable $x$ enters the first singularity at $\phi_{n}$ with a generic value. It loses a degree of freedom, i.e. assumes "fixed" values for the subsequent $N$ steps. If, provided the $p_{n}, q_{n}, r_{n}$ are adequately chosen, it enters the second singularity with precisely the value 0 , it will exit while recovering the lost degree of freedom and thus the singularity is confined [13]. While the Gambier mapping has been studied in this spirit in [8] and [12], the singularity confinement approach need not concern us in what follows. Just as in the continuous case the contiguity relation was derived independently of the Painlevé property, in what follows we shall derive the Schlesinger transformations and the contiguity relation without any reference to the confinement property of the singularities of the mapping.

While we shall not require the confinement property, we shall still, for the derivation of the Schlesinger transformation, work under the assumption $\phi_{n}=\psi_{n+N}$, which introduces naturally the parameter $N$. We must stress here that nothing imposes an integer value for $N$ (and thus no confinement is guaranteed for the mapping). Let us focus first on the $\Delta N=2$ case. We assume that $x$ is generic up to some index $n$, infinite at $n+1$ and becomes fixed from $n+2$ onwards. At the other end, for some other solution, we assume that $x$ is fixed up to $n+N$ where it assumes the value 0 and then becomes generic from $n+N+1$ onwards. For the $x$ obtained through the Schlesinger transformation, denoted by $\bar{x}$, we require that it be generic up to $n-1$, become infinite at $n$ and be fixed from $n+1$ onwards. At the other end, we assume that $\bar{x}$ is fixed up to $n+N+1$ where it assumes the value 0 and becomes generic from $n+N+2$ onwards.

We have thus two singularities situated at a certain distance, which increases by 2 as a consequence of the Schlesinger transformation. Assuming that $x$ becomes infinite at $n+1$ and requiring that $\bar{x}$ become infinite already at $n$ can be guaranteed if the quantity $\left(y-\phi_{n}\right)$ appears in the denominator of $\bar{x}$. At the other end, if we assume that $x$ exits the singularity by being 0 at $n+N$ (and thus 
assuming an indeterminate form $0 / 0$ at $n+N+1$ ) then $\bar{x}$ must become 0 at $n+N+1$ and indeterminate at $n+N+2$. This means that the numerator of $\bar{x}$ must contain the factor $\left(y-\psi_{n-1}\right)$. Putting this together and requiring a $\bar{x}$ homographic in $x$ we arrive at the ansatz

$$
\bar{x}_{n}=\frac{y-\psi_{n-1}}{y-\phi_{n}} \frac{\tilde{\alpha}_{n} x_{n}+\tilde{\beta}_{n}}{\tilde{\gamma}_{n} x_{n}+\tilde{\delta}_{n}}
$$

and require that $\bar{x}$ satisfy the equation

$$
\bar{x}_{n+1}=\frac{\bar{x}_{n}\left(\bar{p}_{n} y+\bar{r}_{n}\right)+\bar{q}_{n}\left(y-\psi_{n-1}\right)}{\bar{x}_{n}\left(y-\phi_{n+1}\right)}
$$

where we have used the fact that $\bar{\phi} \equiv \phi_{n+1}$ and $\bar{\psi} \equiv \psi_{n-1}$.

A straightforward argument allows us to calculate the parameters of (42). Let us assume that $\psi_{n}=y$ and that we have a solution where $x$ is generic up to $n$. In this case, at $n+1$ it will become fixed and takes the value $x_{n+1}=\left(p_{n} \psi_{n}+r_{n}\right) /\left(\psi_{n}-\phi_{n}\right)$. However, from the basic assumption of the Schlesinger, $\bar{x}$ must be generic at $n+1$ an assumption on the surface incompatible with the form of (42), where the numerator vanishes. However this is not the problem. The requirement that $\bar{x}$ be generic at $n+1$ means simply that the denominator of $\bar{x}$ must also vanish, so as to lead to a indeterminate form $0 / 0$, i.e. $\tilde{\gamma}_{n+1} x_{n+1}+\tilde{\delta}_{n+1}=0$ for the value $x_{n+1}$ we just computed. Without loss of generality we can choose $\tilde{\gamma}_{n}=\phi_{n-1}-\psi_{n-1}, \tilde{\delta}_{n}=p_{n-1} \phi_{n-1}+r_{n-1}$. Similarly let us assume that $x_{n+1}$ is generic while $\phi_{n}=y$. Clearly in this case we must have $x_{n}\left(p_{n} \phi_{n}+r_{n}\right)+q_{n}\left(\phi_{n}-\psi_{n}\right)=0$. But for the existence of the Schlesinger we must have a generic $\bar{x}_{n}$ and for this the only possibility is that $\tilde{\alpha}_{n} x_{n}+\tilde{\beta}_{n}=0$ for the $x_{n}$ just obtained. Thus we have $\tilde{\alpha}_{n}=p_{n} \phi_{n}+r_{n}, \tilde{\beta}_{n}=q_{n}\left(\phi_{n}-\psi_{n}\right)$ up to an arbitrary global factor, which, in order to eliminate denominators we have chosen equal to $\left(p_{n-2} \psi_{n-2}+r_{n-2}\right)\left(p_{n-1} \phi_{n-1}+r_{n-1}\right)-q_{n-1} \omega_{n-2} \omega_{n-1}$. The parameters of (43) are now easily obtained. We find,

$$
\begin{aligned}
& \bar{p}_{n}=p_{n}\left(p_{n-1} \psi_{n-1}+r_{n-1}\right)\left(p_{n+1} \phi_{n+1}+r_{n+1}\right)+q_{n+1}\left(p_{n-1} \psi_{n-1}+r_{n-1}\right) \omega_{n+1}-q_{n}\left(p_{n+1} \phi_{n+1}+r_{n+1}\right) \omega_{n-1} \\
& \bar{r}_{n}=r_{n}\left(p_{n-1} \psi_{n-1}+r_{n-1}\right)\left(p_{n+1} \phi_{n+1}+r_{n+1}\right)-\phi_{n} q_{n+1}\left(p_{n-1} \psi_{n-1}+r_{n-1}\right) \omega_{n+1}+\psi_{n} q_{n}\left(p_{n+1} \phi_{n+1}+r_{n+1}\right) \omega_{n-1}
\end{aligned}
$$

$$
\bar{q}_{n}=q_{n}\left(\left(p_{n-2} \psi_{n-2}+r_{n-2}\right)\left(p_{n-1} \phi_{n-1}+r_{n-1}\right)-q_{n-1} \omega_{n-2} \omega_{n-1}\right)\left(\left(p_{n} \psi_{n}+r_{n}\right)\left(p_{n+1} \phi_{n+1}+r_{n+1}\right)-q_{n+1} \omega_{n} \omega_{n+1}\right)
$$

where we have put $\omega_{n}=\phi_{n}-\psi_{n}$.

At this point it is interesting to show that the evolution along the "bar" direction is dual to that along the $n$ direction and thus the Gambier mapping is self-dual in this sense. Indeed, starting from equation (42) we introduce a new variable by $X_{n}=x_{n}+\tilde{\delta}_{n} / \tilde{\gamma}_{n}$ and find an equation of the form

$$
\bar{X}=\frac{X(P y+R)+Q(y-\Psi)}{X(y-\Phi)}
$$

where $\Phi \equiv \phi_{n}, \Psi \equiv \psi_{n-1}$ and $P, Q, R$ are given in terms of the basic parameters, i.e. an equation of the form (41). Similarly, if we introduce the same translated variable into (42) and use the expressions 


\section{A.Ramani, B.Grammaticos and P.Guha}

of $\gamma$ and $\delta$ we find for the Schlesinger transformation

$$
X_{n+1}=\frac{y-\underline{\Psi}}{y-\Phi} \frac{A X_{n}+B}{\Gamma X_{n}+\Delta}
$$

where the $A, B, \Gamma, \Delta$ are expressed in terms of the $p, q, r, \phi, \psi$, i.e. an equation of the same form as (41). Notice that $\underline{\Psi}$ at the numerator of (46) is just $\psi_{n}$.

Having seen that the quantities $\alpha, \beta, \gamma, \delta$ can be expressed in terms of those of the Gambier mapping we can now obtain, formally, the contiguity relation by writing (32) and the equivalent relation in the down-bar direction and eliminating $y$. We find thus

$\bar{x}(\tilde{\gamma} x+\tilde{\delta})\left(x\left(\phi_{n-1}-\phi_{n}\right)\left(\underline{\sim}_{\underline{\alpha}}^{\alpha} \underset{\sim}{\delta}\right)+\omega(\underset{\sim}{\alpha} \underline{x}+\underset{\sim}{\beta})\right)=(\tilde{\alpha} x+\tilde{\beta})\left(x \omega_{n-1}(\underbrace{\alpha}_{\underline{\alpha}}+\underset{\sim}{\delta})+\left(\psi_{n-1}-\psi_{n}\right)(\underset{\sim}{\alpha} \underline{x}+\underset{\sim}{\beta})\right)$

which is precisely a Gambier mapping. In fact the most general Gambier mapping, obtained by equation (39) would have contained 7 arbitrary functions up to a homographic transformation of $x$, which would have reduced the number of functions to just four. Equation (47) is expressed in terms of $p, q, r, \phi, \psi$ up to a gauge in $x$ which would have reduced the number of arbitrary functions to four. Thus we may conclude that (47) is a generic Gambier mapping.

We turn now to the $\Delta N=1$ case. The general idea is the same as that of the case $\Delta N=2$ we examined above. We assume again that $x$ is generic up to some index $n$, infinite at $n+1$ and becomes fixed from $n+2$ onwards. Similarly for the $x$ obtained through the Schlesinger transformation, denoted by $\bar{x}$, we require that it be generic up to $n-1$, become infinite at $n$ and be fixed from $n+1$ onwards. Thus we conclude that $\left(y-\phi_{n}\right)$ must appear in the denominator of $\bar{x}$. We can thus introduce the ansatz

$$
\bar{x}_{n}=\frac{1}{y-\phi_{n}} \frac{\tilde{\alpha}_{n} x_{n}+\tilde{\beta}_{n}}{x_{n}+\tilde{\delta}_{n}}+\tilde{\lambda}_{n}
$$

where the introduction of $\lambda$ will become clear in the following paragraph. If now we assume that $x_{n+1}$ is generic while $\phi_{n}=y$, then $x_{n}$ must be chosen such as $x_{n}\left(p_{n} \phi_{n}+r_{n}\right)+q_{n}\left(\phi_{n}-\psi_{n}\right)=0$. Since for the existence of the Schlesinger we must have a generic $\bar{x}_{n}$, the only possibility is that $\tilde{\alpha}_{n} x_{n}+\tilde{\beta}_{n}=0$ for the $x_{n}$ just obtained. Thus we have $\tilde{\alpha}_{n}=\left(p_{n} \phi_{n}+r_{n}\right) f_{n-1}$ and $\tilde{\beta}_{n}=q_{n}\left(\phi_{n}-\psi_{n}\right) f_{n-1}$ where $f$ is an arbitrary global factor and the shift in the index is more convenient for the subsequent calculations.

We turn now to the other end, where $\psi_{n+N}=y$. At this end we assume that if a singularity exists for $x$, the same singularity will exist at the same point for $\bar{x}$. Thus we ensure that $\Delta N=1$. When the singularity consists in $x_{n+N}$ being generic and $x_{n+N+1}$ fixed the requirement that $\bar{x}$ behaves in the same way is automatically satisfied. So let us now consider the singularity where $x_{n+N}$ becomes 0 and at the next step it recovers a degree of freedom through an indeterminate form $0 / 0$. Requiring that $\bar{x}$ become 0 at the same point leads to the constraint

$$
\tilde{\lambda}_{n}=\frac{\tilde{\beta}_{n}}{\tilde{\delta}_{n}\left(\phi_{n}-\psi_{n}\right)}
$$

Had we not introduced $\lambda$ it would have been impossible to ensure the vanishing of $\bar{x}$. Using the expression of $\tilde{\beta}$ we can obtain simply $\tilde{\lambda}_{n}=-q_{n} f_{n-1} / \tilde{\delta}_{n}$. 
Next we require that $\bar{x}$ satisfy the equation

$$
\bar{x}_{n+1}=\frac{\bar{x}_{n}\left(\bar{p}_{n} y+\bar{r}_{n}\right)+\bar{q}_{n}\left(y-\psi_{n}\right)}{\bar{x}_{n}\left(y-\phi_{n+1}\right)}
$$

where here we have used the fact that $\bar{\phi} \equiv \phi_{n+1}$ but $\bar{\psi} \equiv \psi_{n}$. This allows us to compute the quantities $f, \bar{p}, \bar{q}$ and $\bar{r}$. We find

$$
\begin{gathered}
f_{n}=\tilde{\delta}_{n}\left(p_{n} \phi_{n}+r_{n}\right)+q_{n}\left(\psi_{n}-\phi_{n}\right) \\
\bar{p}_{n}=-\tilde{\delta}_{n} f_{n+1}+\frac{q_{n+1} f_{n}}{\tilde{\delta}_{n+1}} \\
\bar{q}_{n}=q_{n} f_{n+1} f_{n-1} \\
\bar{r}_{n}=\frac{1}{\tilde{\delta}_{n+1}}\left(\tilde{\delta}_{n} f_{n+1}\left(\tilde{\delta}_{n+1} \psi_{n}+p_{n} \psi_{n}+r_{n}\right)-q_{n+1} \psi_{n+1} f_{n}\right)
\end{gathered}
$$

The interesting feature of the $\Delta N=1$ case (compared to the $\Delta N=2$ one) is that here the parameter $\tilde{\delta}$ that appears in the Schlesinger does satisfy a homographic equation

$$
\tilde{\delta}_{n+1} \tilde{\delta}_{n}+p_{n} \tilde{\delta}_{n}-q_{n}=0
$$

involving the parameters of the equation for $x$. It goes without saying that since the two singularities are not treated in the same way the Gambier mapping cannot be self-dual with respect to the transformations induced by the $\Delta N=1$ Schlesinger.

Again the quantities $\alpha, \beta, \delta$ can be expressed in terms of those of the Gambier mapping. Thus the contiguity relation can be obtained formally by writing (48) and the equivalent relation in the down-bar direction and eliminating $y$. We find thus

$$
\frac{(\bar{x}-\tilde{\lambda})(x+\tilde{\delta})}{\tilde{\alpha} x+\tilde{\beta}}-\frac{(x-\underset{\sim}{\lambda})(\underline{x}+\underset{\sim}{\delta})}{\underset{\sim}{\alpha} \underline{x}+\underset{\sim}{\beta}}=\phi_{n}-\phi_{n-1}
$$

again a generic Gambier mapping.

\section{Conclusions}

In this paper we have addressed the question of the existence of contiguity relations for linearisable equations. At first sight a difficulty does exist since for the contiguity relations one needs solutions obtained for values of some parameter differing by an integer value, and it is not clear where a parameter can be inserted given that the linearisable equations contain free functions of the independent variable. In the case of the continuous Gambier equation the dilemma is solved by the fact that this equation is traditionally written in a form containing an explicitly integer parameter. Working with this form we have been able to introduce the Schlesinger transformations. This interesting feature of the Gambier equation is that two different Schlesinger transformations are possible corresponding to steps of the integer parameter $\Delta n=1$ and 2. In both case we were able to derive the contiguity relation in an explicit form.

The discrete Gambier equation presented another challenge since in this case no integer parameter is present. The way to solve this problem was to consider the singularity structure of the system. 


\section{A.Ramani, B.Grammaticos and P.Guha}

Typically a Gambier mapping has two singularities situated at arbitrary values of the independent variable. The essence of a Schlesinger transformation is the introduction of a new variable that would have singularities at points differing by an integer number of steps from the ones of the initial variable. Again, just as in the continuous case, we found that there exist two possibilities corresponding to $\Delta N=1$ and 2 . The contiguity relation in both cases is a Gambier mapping. Moreover in the $\Delta N=2$ case we have shown that the system is self-dual, i.e. the evolution along the independent variable is governed by an equation of the same form when one considers the evolution induced by the Schlesinger transformation along the virtual parameter $N$.

As we have explained in the end of section 2 the parameter $n$ appearing in the Gambier equation does not have to be integer and in fact need not even be a constant. This is an indication that one may be able to introduce parameters in linearisable equations which, if properly chosen, would make possible to lead to Schlesinger-like transformations and thus ultimately to contiguity relations.

\section{Acknowledgments}

P. Guha would like to express his gratitude to IHES for their warm hospitality.

\section{References}

[1] A. Abramowitz and I. Stegun, Handbook of Mathematical Functions, (Dover, 1965).

[2] M. Jimbo and T. Miwa, Physica 2D (1981) 407.

[3] A. Ramani, B. Grammaticos and J. Hietarinta, Phys. Rev. Lett. 67 (1991) 1829.

[4] A. Ramani, Y. Ohta, J. Satsuma and B. Grammaticos, Commun. Math. Phys. 192 (1998) 67.

[5] H. Sakai, Commun. Math. Phys. 220 (2001) 165.

[6] E.L. Ince, Ordinary Differential Equations, (Dover, London, 1956).

[7] A. Ramani, B. Grammaticos and S. Lafortune, Lett. Math. Phys. 46 (1998) 131.

[8] B. Grammaticos and A. Ramani, Physica A 223 (1996) 125.

[9] A. Fokas, B. Grammaticos and A. Ramani, J. of Math. Anal. and Appl. 180 (1993) 342.

[10] J. Hietarinta and C-M. Viallet, Phys. Rev. Lett. 81 (1998) 325.

[11] A. Ramani, B. Grammaticos, S. Lafortune and Y. Ohta, J. Phys. A 33 (2000) L287.

[12] B. Grammaticos, A. Ramani and S. Lafortune Physica A 253 (1998) 260.

[13] B. Grammaticos, A. Ramani and V. Papageorgiou, Phys. Rev. Lett. 67 (1991) 1825. 